\section{Streptokinase equal to TPA}

\section{Washington}

RESUlts from an Italian study comparing the blood-clot dissolving agents tissue plasminogen activator (TPA) and streptokinase show that TPA is no more effective than streptokinase, which is one-tenth of the price and widely used in Europe. Biotechnology industry analysts are worried that sales of the TPA product Activase, which last year brought in $\$ 196.4$ million for the San-Francisco-based Genentech, may be hit by the news.

The study, sponsored by the National Association of Cardiologists and the Mario Negri Institute of Pharmacological Research in Milan, was based on the treatment of 20,891 heart-attack victims.

Results reported last week at a meeting of cardiologists in Florence, are derived from an analysis of only the 12,381 patients involved in the Italian half of the study.

The patients were randomly divided into two groups and given intravenous infusion of either TPA or streptokinase. Half of the patients in each of the two groups also received a subcutaneous injection of heparin 12 hours after administration of the thrombolytic agent. Following standard medical practice, aspirin and beta blockers were also administered. The mortality rates when TPA and streptokinase were given alone were 8.7 and 9.2 per cent, and in combination with heparin the rates were 9.2 and 7.9 per cent. TPA caused fewer allergic reactions, less hypotension and fewer bleeding episodes, but streptokinase patients suffered fewer subsequent strokes.
For reasons of cost alone, these findings could significantly alter US medical practice, which at present favours TPA.

Genentech is the only US company with approval to sell a recombinant version of TPA, and its greatest competition comes from streptokinase, which is derived from streptococcal bacteria and sold in the United States by Hoechst-Roussel Pharmaceuticals and SmithKline Beecham. Activase, which costs $\$ 2,200$ per dose, now commands a two-thirds share of the US thrombolytic market.

Genentech claims that the significance of the Italian study may be reduced because it did not use the treatment favoured by "98 per cent of US cardiologists", whereby intravenous heparin is given concurrently with the thrombolytic agent.

San Francisco stock analyst John Girton says the results of the trial make it "harder to justify the price of TPA", but he believes that TPA is still a healthy proposition, and points out that it may have other uses. Indeed, Genentech is awaiting approval of Activase for pulmonary embolism, and is conducting clinical trials for the treatment of both unstable angina and occlusive stroke.

Girton does not foresee any impact on the Genentech agreement, where Roche will acquire a 60 per cent stake in Genentech in return for a $\$ 2,100$-million investment. But Stuart Weisbrod of PrudentialBache takes a gloomier view and predicts that the growth in sales of Activase will slow from 25 to 30 per cent a year to 5 per cent.

Diane Gershon
AIDS drug
trial resumes

\section{Washington}

THE controversial study of the AIDS drug Compound $\mathrm{Q}$, which was halted last August by the US Food and Drug Administration (FDA), is to be resumed with full FDA approval. It is the first time the FDA has granted an independent IND (Investigational New Drug) licence to a community-based research group.

News of the decision will enrage many doctors and researchers who feel that the original 'clandestine' study, coordinated by the San Francisco-based advocacy group Project Inform, was poorly designed, gave inconclusive results and may have caused unnecessary suffering to patients (Nature 341, 267; 28 September 1989). The ad hoc study began last May after in vitro experiments showed that Compound $\mathrm{Q}$, a protein (trichosanthin) derived from a Chinese cucumber-like plant, selectively killed cells infected with HIV.

Sandoz Pharmaceuticals of East Hanover, New Jersey is providing Project Inform with $\$ 250,000$ in financial aid, stipulating that the grant is for the retreatment of patients who participated in the unauthorized study last year. In addition, Genelabs of Redwood City, California is donating a highly purified form of Compound Q called GLQ223.

The FDA's decision to approve the trial has caused Donald Abrams, an AIDS researcher at the San Francisco General Hospital, to reconsider his position on the agency's antiviral drugs advisory committee. He thinks that the FDA should have consulted with the advisory committee in view of the atypical nature of the new clinical trial and the controversy that surrounds the original unauthorized trial. He believes it sets a bad precedent, as the same people who performed an unauthorized trial have now received approval from FDA.

The new study is designed to evaluate the safety and effectiveness of GLQ223 over the longer term. GLQ223 will be administered to over 100 patients in San Francisco, Los Angeles, Miami and Florida, all of whom will have previously been treated with the Chinese version of the drug. Patients will be assigned randomly to two groups, one receiving treatment every three weeks, the other every six weeks, while being allowed to continue with other AIDS therapies.

Genelabs, who holds the patent rights for GLQ223 as a possible treatment for HIV infection, ARC and AIDS, is evaluating the drug in its own FDA-approved clinical trials. Preliminary findings of phase I trials are expected to be submitted for FDA review in mid-1990.

Diane Gershon 